\title{
Study of $e^{+} e^{-} \rightarrow 2(p \bar{p})$ at center-of-mass energies between 4.0 and 4.6 GeV
}

M. Ablikim, ${ }^{1}$ M. N. Achasov, ${ }^{10, c}$ P. Adlarson,${ }^{67}$ S. Ahmed, ${ }^{15}$ M. Albrecht, ${ }^{4}$ R. Aliberti, ${ }^{28}$ A. Amoroso, ${ }^{66 a, 66 c}$ Q. An,${ }^{63,50}$ Anita, ${ }^{21}$ X. H. Bai,${ }^{57}$ Y. Bai, ${ }^{49}$ O. Bakina,${ }^{29}$ R. Baldini Ferroli, ${ }^{23 a}$ I. Balossino, ${ }^{24 a}$ Y. Ban,${ }^{39, k}$ K. Begzsuren,${ }^{26}$ N. Berger,${ }^{28}$ M. Bertani, ${ }^{23 a}$ D. Bettoni, ${ }^{24 a}$ F. Bianchi, ${ }^{66 a, 66 c}$ J. Biernat ${ }^{67} \mathrm{~J}$. Bloms, ${ }^{60}$ A. Bortone,${ }^{66 a, 66 \mathrm{c}}$ I. Boyko, ${ }^{29}$ R. A. Briere, ${ }^{5}$ H. Cai ${ }^{68}$ X. Cai, ${ }^{1,50}$ A. Calcaterra, ${ }^{23 a}$ G. F. Cao, ${ }^{1,55}$ N. Cao, ${ }^{1,55}$ S. A. Cetin, ${ }^{54 a}$ J. F. Chang, ${ }^{1,50}$ W. L. Chang, ${ }^{1,55}$ G. Chelkov, ${ }^{29, b}$ D. Y. Chen, ${ }^{6}$ G. Chen, ${ }^{1}$ H. S. Chen, ${ }^{1,55}$ M. L. Chen, ${ }^{1,50}$ S. J. Chen,${ }^{36}$ X. R. Chen, ${ }^{25}$ Y. B. Chen, ${ }^{1,50}$ Z. J. Chen, ${ }^{20,1}$ W. S. Cheng, ${ }^{66 c}$ G. Cibinetto, ${ }^{24 a}$ F. Cossio, ${ }^{66 c}$ X. F. Cui, ${ }^{37}$ H. L. Dai, ${ }^{1,50}$ X. C. Dai, ${ }^{1,55}$ A. Dbeyssi, ${ }^{15}$ R. B. de Boer, ${ }^{4}$ D. Dedovich, ${ }^{29}$ Z. Y. Deng, ${ }^{1}$ A. Denig, ${ }^{28}$ I. Denysenko ${ }^{29}$ M. Destefanis, ${ }^{66 a, 66 c}$ F. De Mori, ${ }^{66 a, 66 c}$ Y. Ding, ${ }^{34}$ C. Dong, ${ }^{37}$ J. Dong, ${ }^{1,50}$ L. Y. Dong, ${ }^{1,55}$ M. Y. Dong, ${ }^{1,50,55}$ X. Dong ${ }^{68}$ S. X. Du, ${ }^{71}$ J. Fang, ${ }^{1,50}$ S. S. Fang, ${ }^{1,55}$ Y. Fang, ${ }^{1}$ R. Farinelli, ${ }^{24 a}$ L. Fava, ${ }^{66 b, 66 c}$ F. Feldbauer, ${ }^{4}$ G. Felici, ${ }^{23 a}$ C. Q. Feng, ${ }^{63,50}$ M. Fritsch, ${ }^{4}$ C. D. Fu, ${ }^{1}$ Y. Fu, ${ }^{1}$ Y. Gao,${ }^{39, k}$ Y. Gao ${ }^{64}$ Y. Gao,${ }^{63,50}$ Y. G. Gao, ${ }^{6}$ I. Garzia, ${ }^{24 a, 24 b}$ E. M. Gersabeck, ${ }^{58}$ A. Gilman, ${ }^{59}$ K. Goetzen, ${ }^{11}$ L. Gong, ${ }^{34}$ W. X. Gong, ${ }^{1,50}$ W. Gradl, ${ }^{28}$ M. Greco, ${ }^{66 a, 66 c}$ L. M. Gu, ${ }^{36}$ M. H. Gu, ${ }^{1,50}$ S. Gu, ${ }^{2}$ Y. T. Gu, ${ }^{13}$ C. Y. Guan, ${ }^{1,55}$ A. Q. Guo, ${ }^{22}$ L. B. Guo, ${ }^{35}$ R. P. Guo, ${ }^{41}$ Y. P. Guo, ${ }^{9, h}$ Y. P. Guo,${ }^{28}$ A. Guskov,${ }^{29}$ T. T. Han,${ }^{42}$ X. Q. Hao,${ }^{16}$ F. A. Harris,${ }^{56}$ K. L. He, ${ }^{1,55}$ F. H. Heinsius, ${ }^{4}$ C. H. Heinz, ${ }^{28}$

T. Held, ${ }^{4}$ Y. K. Heng, ${ }^{1,50,55}$ C. Herold, ${ }^{52}$ M. Himmelreich, ${ }^{11, f}$ T. Holtmann, ${ }^{4}$ Y. R. Hou, ${ }^{55}$ Z. L. Hou, ${ }^{1}$ H. M. Hu, ${ }^{1,55}$ J. F. Hu, ${ }^{48, m}$ T. Hu,${ }^{1,50,55}$ Y. Hu, ${ }^{1}$ G. S. Huang, ${ }^{63,50}$ L. Q. Huang,${ }^{64}$ X. T. Huang, ${ }^{42}$ Y. P. Huang, ${ }^{1}$ Z. Huang, ${ }^{39, k}$ N. Huesken,${ }^{60}$ T. Hussain, ${ }^{65}$ W. Ikegami Andersson, ${ }^{67}$ W. Imoehl, ${ }^{22}$ M. Irshad, ${ }^{63,50}$ S. Jaeger, ${ }^{4}$ S. Janchiv, ${ }^{26, j}$ Q. Ji, ${ }^{1}$ Q. P. Ji, ${ }^{16}$ X. B. Ji,,${ }^{1,55}$ X. L. Ji, ${ }^{1,50}$ H. B. Jiang, ${ }^{42}$ X. S. Jiang, ${ }^{1,50,55}$ X. Y. Jiang, ${ }^{37}$ J. B. Jiao, ${ }^{42}$ Z. Jiao, ${ }^{18}$ S. Jin ${ }^{36}$ Y. Jin, ${ }^{57}$ T. Johansson, ${ }^{67}$ N. Kalantar-Nayestanaki, ${ }^{31}$ X. S. Kang, ${ }^{34}$ R. Kappert, ${ }^{31}$ M. Kavatsyuk, ${ }^{31}$ B. C. Ke, ${ }^{44,1}$ I. K. Keshk, ${ }^{4}$ A. Khoukaz, ${ }^{60}$ P. Kiese, ${ }^{28}$ R. Kiuchi, ${ }^{1}$ R. Kliemt, ${ }^{11}$ L. Koch, ${ }^{30}$ O. B. Kolcu, ${ }^{54 a, e}$ B. Kopf, ${ }^{4}$ M. Kuemmel, ${ }^{4}$ M. Kuessner, ${ }^{4}$ A. Kupsc, ${ }^{67}$ M. G. Kurth, ${ }^{1,55}$ W. Kühn, ${ }^{30}$ J. J. Lane, ${ }^{58}$ J. S. Lange, ${ }^{30}$ P. Larin, ${ }^{15}$ L. Lavezzi, ${ }^{66,66 c}$ Z. H. Lei, ${ }^{63,50}$ H. Leithoff, ${ }^{28}$ M. Lellmann, ${ }^{28}$ T. Lenz, ${ }^{28}$ C. Li ${ }^{40}$ C. H. Li, ${ }^{33}$ Cheng Li, ${ }^{63,50}$ D. M. Li ${ }^{71}$ F. Li, ${ }^{1,50}{ }^{3}$. Li, ${ }^{1}$ H. Li $,{ }^{44} \mathrm{H} . \mathrm{Li},{ }^{63,50} \mathrm{H} . \mathrm{B} . \mathrm{Li}^{1,55}$

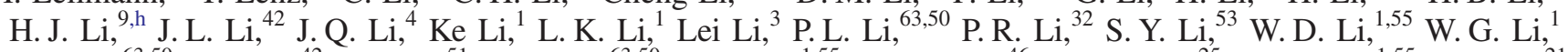
X. H. Li ${ }^{63,50}$ X. L. Li ${ }^{42}$ Z. Y. Li ${ }^{51}$ H. Liang,${ }^{63,50}$ H. Liang, ${ }^{1,55}$ Y. F. Liang, ${ }^{46}$ Y. T. Liang, ${ }^{25}$ L. Z. Liao, ${ }^{1,55}$ J. Libby, ${ }^{21}$ C. X. Lin, ${ }^{51}$ B. J. Liu, ${ }^{1}$ C. X. Liu, ${ }^{1}$ D. Liu ${ }^{63,50}{ }^{\text {F. H. Liu }}{ }^{45}$ Fang Liu, ${ }^{1}$ Feng Liu, ${ }^{6}$ H. B. Liu, ${ }^{13}{ }^{3}$ H. M. Liu,,${ }^{1,55}$ Huanhuan Liu, ${ }^{1}$ Huihui Liu, ${ }^{17}$ J. B. Liu ${ }^{63,50}$ J. Y. Liu ${ }^{1,55}$ K. Liu, ${ }^{1}$ K. Y. Liu, ${ }^{34}$ Ke Liu, ${ }^{6}$ L. Liu, ${ }^{63,50}$ M. H. Liu, ${ }^{9, h}$ Q. Liu ${ }^{55}$ S. B. Liu, ${ }^{63,50}$ Shuai Liu, ${ }^{47}$ T. Liu, ${ }^{1,55}$ W. M. Liu ${ }^{63,50}$ X. Liu, ${ }^{32}$ Y. B. Liu, ${ }^{37}$ Z. A. Liu, ${ }^{1,50,55}$ Z. Q. Liu, ${ }^{42}$ X. C. Lou, ${ }^{1,50,55}$ F. X. Lu, ${ }^{16}$ H. J. Lu, ${ }^{18}$ J. D. Lu, ${ }^{1,55}$ J. G. Lu, ${ }^{1,50}$ X. L. Lu, ${ }^{1}$ Y. Lu, ${ }^{1}$ Y. P. Lu, ${ }^{1,50}$ C. L. Luo, ${ }^{35}$ M. X. Luo, ${ }^{70}$ P. W. Luo, ${ }^{51}$ T. Luo, X. L. Luo, ${ }^{1,50}$ S. Lusso, ${ }^{66 c}$ X. R. Lyu, ${ }^{55}$ F. C. Ma, ${ }^{34}$ H. L. Ma, ${ }^{1}$ L. L. Ma, ${ }^{42}$ M. M. Ma, ${ }^{1,55}$ Q. M. Ma, ${ }^{1}$ R. Q. Ma, ${ }^{1,55}$ R. T. Ma, ${ }^{55}$ X. N. Ma, ${ }^{37}$ X. X. Ma, ${ }^{1,55}$ X. Y. Ma,${ }^{1,50}$ F. E. Maas, ${ }^{15}$ M. Maggiora, ${ }^{66 a, 66 c}$ S. Maldaner, ${ }^{28}$ S. Malde, ${ }^{61}$ Q. A. Malik, ${ }^{65}$ A. Mangoni, ${ }^{23 b}$ Y. J. Mao, ${ }^{39, k}$ Z. P. Mao, ${ }^{1}$ S. Marcello, ${ }^{66 a, 66 c}$ Z. X. Meng, ${ }^{57}$ J. G. Messchendorp, ${ }^{31}$ G. Mezzadri, ${ }^{24 a}$ T. J. Min, ${ }^{36}$ R. E. Mitchell, ${ }^{22}$ X. H. Mo, ${ }^{1,50,55}$ Y. J. Mo, ${ }^{6}$ N. Yu. Muchnoi, ${ }^{10, c}$ H. Muramatsu, ${ }^{59}$ S. Nakhoul, ${ }^{11, \mathrm{f}}$ Y. Nefedov, ${ }^{29}$ F. Nerling, ${ }^{11, \mathrm{f}}$ I. B. Nikolaev, ${ }^{10, \mathrm{c}}$ Z. Ning, ${ }^{1,50}$ S. Nisar, ${ }^{8, \mathrm{i}}$ S. L. Olsen, ${ }^{55}$ Q. Ouyang, ${ }^{1,50,55}$ S. Pacetti, ${ }^{23 b, 23 c}$ X. Pan,,${ }^{9, h}$ Y. Pan, ${ }^{58}$ A. Pathak, ${ }^{1}$ P. Patteri, ${ }^{23 a}$ M. Pelizaeus ${ }^{4}$ H. P. Peng, ${ }^{63,50}$ K. Peters, ${ }^{11, f}$ J. Pettersson, ${ }^{67}$ J. L. Ping, ${ }^{35}$ R. G. Ping, ${ }^{1,55}$ A. Pitka, ${ }^{4}$ R. Poling, ${ }^{59}$ V. Prasad, ${ }^{63,50}$ H. Qi,${ }^{6,50}$ H. R. Qi ${ }^{53}$ K. H. Qi, ${ }^{25}$ M. Qi ${ }^{36}$ T. Y. Qi,${ }^{9}$ T. Y. Qi, ${ }^{2}$ S. Qian, ${ }^{1,50}$ W.-B. Qian ${ }^{55}$ Z. Qian, ${ }^{51}$ C. F. Qiao, ${ }^{55}$ L. Q. Qin, ${ }^{12}$ X. S. Qin, ${ }^{4}$ Z. H. Qin, ${ }^{1,50}$ J. F. Qiu, ${ }^{1}$ S. Q. Qu ${ }^{37}$ K. H. Rashid, ${ }^{65}$ K. Ravindran, ${ }^{21}$ C. F. Redmer, ${ }^{28}$ A. Rivetti, ${ }^{66 c}$ V. Rodin, ${ }^{31}$ M. Rolo, ${ }^{66 c}$ G. Rong, ${ }_{1,55}$ Ch. Rosner, ${ }^{15}$ M. Rump ${ }^{60}$ H. S. Sang, ${ }^{63}$ A. Sarantsev, ${ }^{29, d}$ Y. Schelhaas, ${ }^{28}$ C. Schnier, ${ }^{4}$ K. Schoenning, ${ }^{67}$ M. Scodeggio, ${ }^{24 a}$ D. C. Shan, ${ }^{47}$ W. Shan, ${ }^{19}$ X. Y. Shan, ${ }^{63,50}$ M. Shao, ${ }^{63,50}$ C. P. Shen, ${ }^{9}$ P. X. Shen, ${ }^{37}$ X. Y. Shen, ${ }^{1,55}$ H. C. Shi, ${ }^{63,50}$ R. S. Shi, ${ }^{1,55}$ X. Shi, ${ }^{1,50}$ X. D. Shi ${ }^{63,50}$ W. M. Song, ${ }^{27,1}$ Y. X. Song, ${ }^{39, \mathrm{k}}$ S. Sosio, ${ }^{66,66 \mathrm{c}}$ S. Spataro, ${ }^{66,66 \mathrm{c}}$ K. X. Su ${ }^{68}$ F. F. Sui, ${ }^{42}$ G. X. Sun, ${ }^{1}$ H. K. Sun, ${ }_{1}$ J. F. Sun, ${ }^{16}$ L. Sun, ${ }^{68}$ S. S. Sun, ${ }^{1,55}$ T. Sun, ${ }^{1,55}$ W. Y. Sun, ${ }^{35}$ X. Sun, ${ }^{20,1}$ Y. J. Sun,${ }^{63,50}$ Y. K. Sun ${ }^{63,50}$ Y.Z. Sun, ${ }^{1}$ Z. T. Sun ${ }^{1}$ Y. H. Tan, ${ }^{68}$ Y. X. Tan, ${ }^{63,50}$ C. J. Tang, ${ }^{46}$ G. Y. Tang,,${ }^{1}$ J. Tang, ${ }^{51}$ J. X. Teng, ${ }^{63,50}$ V. Thoren, ${ }^{67}$ I. Uman,${ }^{54 b}$ C. W. Wang ${ }^{36}$ D. Y. Wang, ${ }^{39, k}$ H. P. Wang ${ }^{1,55}$ K. Wang, ${ }_{63,50}^{1,50}$ L. L. Wang, ${ }_{9,}^{1}$ M. Wang, ${ }^{42}$ M. Z. Wang, ${ }^{39, k}$ Meng Wang, ${ }^{1,55}$ W. H. Wang ${ }^{68}$ W. P. Wang, ${ }^{63,50}$ X. Wang, ${ }^{39, k}$ X. F. Wang, ${ }^{32}$ X. L. Wang, ${ }^{9, h}$ Y. Wang, ${ }^{51}$ Y. Wang, ${ }^{63,50}$ Y. D. Wang, ${ }^{38}$ Y. F. Wang, ${ }^{1,50,55}$ Y. Q. Wang, ${ }^{1}$ Z. Wang, ${ }_{58}^{1,50}$ Z. Y. Wang, ${ }^{1}$ Ziyi Wang, ${ }^{55}$ Zongyuan Wang, ${ }^{1,55}$ D. H. Wei, ${ }^{12}$ P. Weidenkaff, ${ }^{28}$ F. Weidner ${ }^{60}$ S. P. Wen, ${ }^{1}$ D. J. White, ${ }^{58}$ U. Wiedner, ${ }^{4}$ G. Wilkinson, ${ }^{61}$ M. Wolke, ${ }^{67}$ L. Wollenberg, ${ }^{4}$ J. F. Wu, ${ }^{1,55}$ L. H. Wu, ${ }^{1}{ }^{1}$ L. J. Wu, ${ }^{1,55}$ X. Wu, ${ }^{9, h}$ Z. Wu, ${ }^{1,50}$ L. Xia,${ }^{63,50}$ H. Xiao, ${ }^{9, h}$ S. Y. Xiao, ${ }^{1}$ Y. J. Xiao, ${ }^{1,55}$ Z. J. Xiao, ${ }^{35}$ X. H. Xie, ${ }^{39, k}$ Y. G. Xie, ${ }^{1,50}$ Y. H. Xie, ${ }^{6}$ T. Y. Xing, ${ }^{1,55}$ G. F. Xu, ${ }^{1}$ J. J. Xu, ${ }^{66}$ Q. J. Xu, ${ }^{14}$ W. Xu, ${ }^{1,55}$ X. P. Xu, ${ }^{47}$ F. Yan, ${ }^{9, h}$ L. Yan, ${ }^{66 a, 66 c}$ L. Yan,,${ }^{9, h}$ W. B. Yan, ${ }^{63,50}$ W. C. Yan, ${ }^{71}$ Xu Yan, ${ }^{47}$ H. J. Yang, ${ }^{43,9}$ H. X. Yang, ${ }^{1}$ L. Yang, ${ }^{44}$ R. X. Yang, ${ }^{63,50}$ S. L. Yang, ${ }^{55}$ S. L. Yang, ${ }^{1,55}$ Y. H. Yang, ${ }^{36}$ Y.X. Yang, ${ }^{12}$ Yifan Yang, ${ }^{1,55}$ Zhi Yang, ${ }^{25}$ M. Ye, ${ }^{1,50}$ M. H. Ye, ${ }^{7}$ J. H. Yin, ${ }^{1}$ Z. Y. You, ${ }^{51}$ B. X. Yu,${ }^{1,50,55}$ C. X. Yu, ${ }^{37}$ G. Yu, ${ }^{1,55}$ J. S. Yu, ${ }^{20,1}$ T. Yu, ${ }^{64}$ C.Z. Yuan, ${ }^{1,55}$ L. Yuan, ${ }^{2}$ W. Yuan, ${ }^{66,66 c}$ X. Q. Yuan, ${ }^{39, k}$ Y. Yuan, ${ }^{1}$ Z. Y. Yuan, ${ }^{51}$ C. X. Yue, ${ }^{33}$ A. Yuncu, ${ }^{54 a, a}$ A. A. Zafar, ${ }^{65}$ Y. Zeng, ${ }^{20,1}$ B. X. Zhang, ${ }^{1}$ Guangyi Zhang, ${ }^{16}$ H. Zhang, ${ }^{63}$ H. H. Zhang, ${ }^{51}$ H. Y. Zhang, ${ }^{1,50}$ 
J. J. Zhang, ${ }^{44}$ J. L. Zhang, ${ }^{69}$ J. Q. Zhang, ${ }^{4}$ J. W. Zhang, ${ }^{1,50,55}$ J. Y. Zhang, ${ }^{1}$ J. Z. Zhang, ${ }^{1,55}$ Jianyu Zhang, ${ }^{1,55}$ Jiawei Zhang, ${ }_{1,55}$ Lei Zhang, ${ }^{36}$ S. Zhang, ${ }^{51}$ S. F. Zhang, ${ }^{36}$ Shulei Zhang, ${ }^{20,1}$ X. D. Zhang, ${ }^{38}$ X. Y. Zhang, ${ }^{42}$ Y. Zhang, ${ }^{61}$ Y. H. Zhang, ${ }^{1,50}$ Y. T. Zhang, ${ }^{63,50}$ Yan Zhang, ${ }^{63,50}$ Yao Zhang, ${ }^{1}$ Yi Zhang, ${ }^{9}$ h Z. H. Zhang, ${ }^{6}$ Z. Y. Zhang, ${ }^{68}$ G. Zhao, ${ }_{1}$ J. Zhao, ${ }^{33}$ J. Y. Zhao, ${ }^{1,55}$ J. Z. Zhao, ${ }_{1,50}$ Lei Zhao, ${ }^{63,50}$ Ling Zhao, ${ }^{1}$ M. G. Zhao, ${ }^{37}$ Q. Zhao, ${ }_{1}$ S. J. Zhao, ${ }^{71}$ Y. B. Zhao, ${ }^{1,50}$ Y. X. Zhao, ${ }^{25}$ Z. G. Zhao, ${ }^{63,50}$ A. Zhemchugov, ${ }^{29, b}$ B. Zheng, ${ }^{64}$ J. P. Zheng, ${ }^{1,50}$ Y. Zheng, ${ }^{39, k}$ Y. H. Zheng, ${ }^{55}$ B. Zhong, ${ }^{35}$ C. Zhong, ${ }^{64}$ L. P. Zhou, ${ }^{1,55}$ Q. Zhou, ${ }^{1,55}$ X. Zhou ${ }^{68}$ X. K. Zhou, ${ }^{55}$ X. R. Zhou, ${ }^{63,50}$ A. N. Zhu, ${ }^{1,55}$ J. Zhu, ${ }^{37}$ K. Zhu, ${ }^{1}$ K. J. Zhu, ${ }^{1,50,55}$ S. H. Zhu, ${ }^{62}$ T. J. Zhu, ${ }^{69}$ W. J. Zhu, ${ }^{37}$ X. L. Zhu, ${ }^{53}$ Y. C. Zhu, ${ }^{63,50}$ Z. A. Zhu, ${ }^{1,55}$ B. S. Zou, ${ }^{1}$ and J. H. Zou ${ }^{1}$

\title{
(BESIII Collaboration)
}

\author{
${ }^{1}$ Institute of High Energy Physics, Beijing 100049, People's Republic of China \\ ${ }^{2}$ Beihang University, Beijing 100191, People's Republic of China \\ ${ }^{3}$ Beijing Institute of Petrochemical Technology, Beijing 102617, People's Republic of China \\ ${ }^{4}$ Bochum Ruhr-University, D-44780 Bochum, Germany \\ ${ }^{5}$ Carnegie Mellon University, Pittsburgh, Pennsylvania 15213, USA \\ ${ }^{6}$ Central China Normal University, Wuhan 430079, People's Republic of China \\ ${ }^{7}$ China Center of Advanced Science and Technology, Beijing 100190, People's Republic of China \\ ${ }^{8}$ COMSATS University Islamabad, Lahore Campus, Defence Road, \\ Off Raiwind Road, 54000 Lahore, Pakistan \\ ${ }^{9}$ Fudan University, Shanghai 200443, People's Republic of China \\ ${ }^{10}$ G.I. Budker Institute of Nuclear Physics SB RAS (BINP), Novosibirsk 630090, Russia \\ ${ }^{11}$ GSI Helmholtzcentre for Heavy Ion Research GmbH, D-64291 Darmstadt, Germany \\ ${ }^{12}$ Guangxi Normal University, Guilin 541004, People's Republic of China \\ ${ }^{13}$ Guangxi University, Nanning 530004, People's Republic of China \\ ${ }^{14}$ Hangzhou Normal University, Hangzhou 310036, People's Republic of China \\ ${ }^{15}$ Helmholtz Institute Mainz, Johann-Joachim-Becher-Weg 45, D-55099 Mainz, Germany \\ ${ }^{16}$ Henan Normal University, Xinxiang 453007, People's Republic of China \\ ${ }^{17}$ Henan University of Science and Technology, Luoyang 471003, People's Republic of China \\ ${ }^{18}$ Huangshan College, Huangshan 245000, People's Republic of China \\ ${ }^{19}$ Hunan Normal University, Changsha 410081, People's Republic of China \\ ${ }^{20}$ Hunan University, Changsha 410082, People's Republic of China \\ ${ }^{21}$ Indian Institute of Technology Madras, Chennai 600036, India \\ ${ }^{22}$ Indiana University, Bloomington, Indiana 47405, USA \\ ${ }^{23 a}$ INFN Laboratori Nazionali di Frascati, I-00044, Frascati, Italy \\ ${ }^{23 \mathrm{~b}}$ INFN Sezione di Perugia, I-06100, Perugia, Italy \\ ${ }^{230}$ University of Perugia, I-06100, Perugia, Italy \\ ${ }^{24 a}$ INFN Sezione di Ferrara, I-44122, Ferrara, Italy \\ ${ }^{24 \mathrm{~b}}$ University of Ferrara, I-44122, Ferrara, Italy \\ ${ }^{25}$ Institute of Modern Physics, Lanzhou 730000, People's Republic of China \\ ${ }^{26}$ Institute of Physics and Technology, Peace Avenue 54B, Ulaanbaatar 13330, Mongolia \\ ${ }^{27}$ Jilin University, Changchun 130012, People's Republic of China \\ ${ }^{28}$ Johannes Gutenberg University of Mainz, Johann-Joachim-Becher-Weg 45, D-55099 Mainz, Germany \\ ${ }^{29}$ Joint Institute for Nuclear Research, 141980 Dubna, Moscow region, Russia \\ ${ }^{30}$ Justus-Liebig-Universitaet Giessen, II. Physikalisches Institut, \\ Heinrich-Buff-Ring 16, D-35392 Giessen, Germany \\ ${ }^{31}$ KVI-CART, University of Groningen, NL-9747 AA Groningen, The Netherlands \\ ${ }^{32}$ Lanzhou University, Lanzhou 730000, People's Republic of China \\ ${ }^{33}$ Liaoning Normal University, Dalian 116029, People's Republic of China \\ ${ }^{34}$ Liaoning University, Shenyang 110036, People's Republic of China \\ ${ }^{35}$ Nanjing Normal University, Nanjing 210023, People's Republic of China \\ ${ }^{36}$ Nanjing University, Nanjing 210093, People's Republic of China \\ ${ }^{37}$ Nankai University, Tianjin 300071, People's Republic of China \\ ${ }^{38}$ North China Electric Power University, Beijing 102206, People's Republic of China \\ ${ }^{39}$ Peking University, Beijing 100871, People's Republic of China \\ ${ }^{40}$ Qufu Normal University, Qufu 273165, People's Republic of China \\ ${ }^{41}$ Shandong Normal University, Jinan 250014, People's Republic of China \\ ${ }^{42}$ Shandong University, Jinan 250100, People's Republic of China \\ ${ }^{43}$ Shanghai Jiao Tong University, Shanghai 200240, People's Republic of China
}




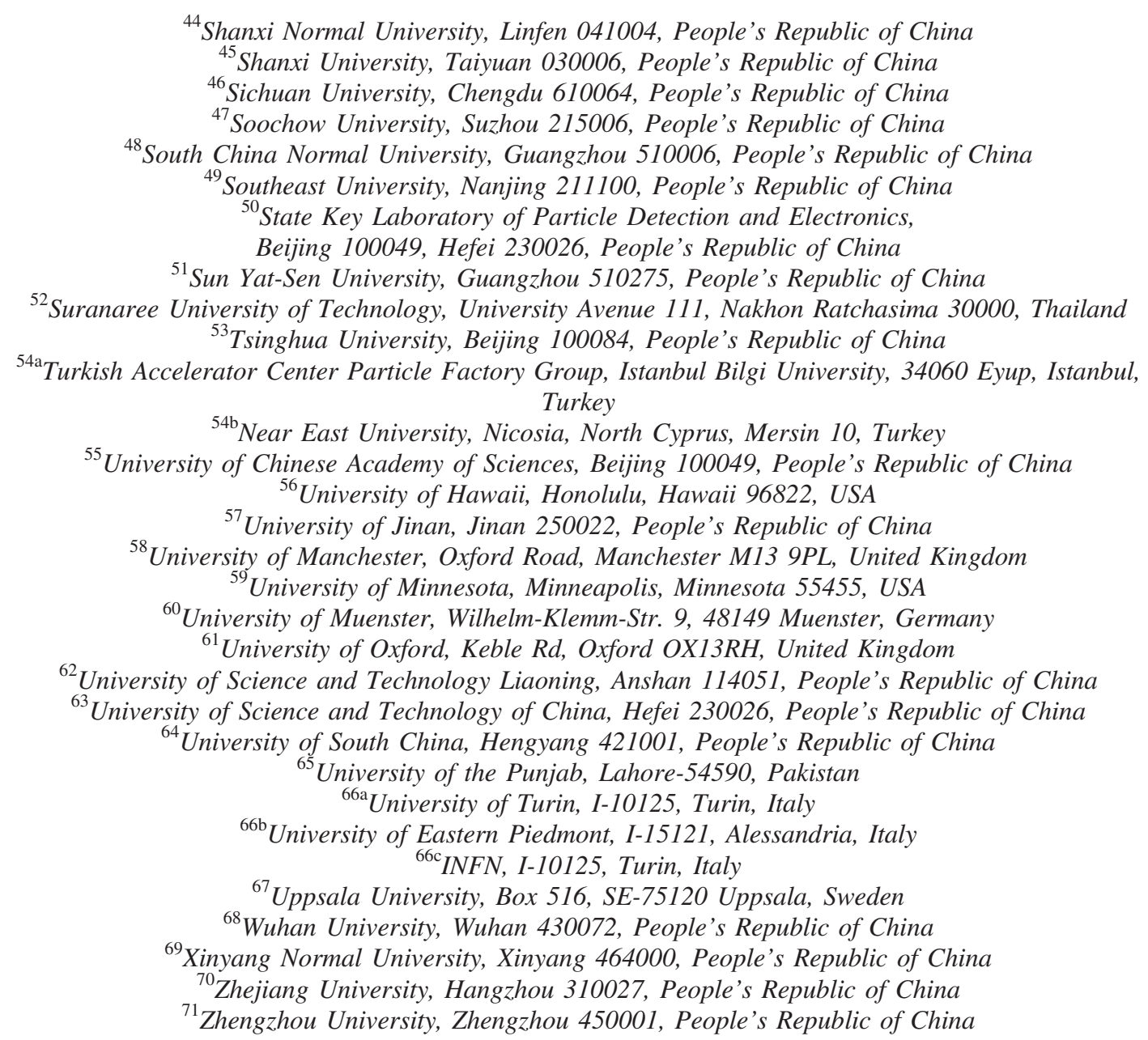

(Received 21 December 2020; accepted 21 January 2021; published 12 March 2021)

Using data taken at 23 center-of-mass energies between 4.0 and $4.6 \mathrm{GeV}$ with the BESIII detector at the Beijing Electron Positron Collider and with a total integrated luminosity of approximately $15 \mathrm{fb}^{-1}$, the process $e^{+} e^{-} \rightarrow 2(p \bar{p})$ is studied for the first time. The Born cross sections for $e^{+} e^{-} \rightarrow 2(p \bar{p})$ are

\footnotetext{
${ }^{a}$ Also at Bogazici University, 34342 Istanbul, Turkey.

${ }^{\mathrm{b}}$ Also at the Moscow Institute of Physics and Technology, Moscow 141700, Russia.

'Also at the Novosibirsk State University, Novosibirsk 630090, Russia.

"Also at the NRC "Kurchatov Institute", PNPI, Gatchina 188300, Russia.

${ }^{\mathrm{e} A l s o}$ at Istanbul Arel University, 34295 Istanbul, Turkey.

${ }^{\mathrm{f}}$ Also at Goethe University Frankfurt, 60323 Frankfurt am Main, Germany.

${ }^{\mathrm{g}}$ Also at Key Laboratory for Particle Physics, Astrophysics and Cosmology, Ministry of Education; Shanghai Key Laboratory for Particle Physics and Cosmology; Institute of Nuclear and Particle Physics, Shanghai 200240, People's Republic of China.

${ }^{\mathrm{h}}$ Also at Key Laboratory of Nuclear Physics and Ion-beam Application (MOE) and Institute of Modern Physics, Fudan University, Shanghai 200443, People's Republic of China.

${ }^{i}$ Also at Harvard University, Department of Physics, Cambridge, Massachusetts 02138, USA.

${ }_{\mathrm{j}}^{\mathrm{j}}$ Present address: Institute of Physics and Technology, Peace Ave.54B, Ulaanbaatar 13330, Mongolia.

${ }^{k}$ Also at State Key Laboratory of Nuclear Physics and Technology, Peking University, Beijing 100871, People's Republic of China. ${ }^{1}$ School of Physics and Electronics, Hunan University, Changsha 410082, China.

${ }^{\mathrm{m}}$ Also at Guangdong Provincial Key Laboratory of Nuclear Science, Institute of Quantum Matter, South China Normal University, Guangzhou 510006, China.

Published by the American Physical Society under the terms of the Creative Commons Attribution 4.0 International license. Further distribution of this work must maintain attribution to the author(s) and the published article's title, journal citation, and DOI. Funded by SCOAP.
} 
measured, and no significant structure is observed in the lineshape. The baryon pair $(p p$ and $\bar{p} \bar{p})$ invariant mass spectra are consistent with phase space, therefore no hexaquark or di-baryon state is found.

DOI: 10.1103/PhysRevD.103.052003

\section{INTRODUCTION}

Since 2003, a series of charmonium-like states, such as $X(3872)$ [1], $Y(4260)$ [2], and $Z_{c}(3900)$ [3,4], were discovered. $Y(4260)$ was first observed by the BABAR experiment via the initial-state radiative (ISR) process $e^{+} e^{-} \rightarrow \gamma_{\mathrm{ISR}} \pi^{+} \pi^{-} J / \psi$, and was confirmed by the CLEO [5] and Belle experiments [6]. In 2017, BESIII reported precise measurements of the $e^{+} e^{-} \rightarrow \pi^{+} \pi^{-} J / \psi$ cross sections in the energy region between 3.77 and $4.60 \mathrm{GeV}$ [7]. Two structures were observed with masses of $4222.0 \pm$ $3.1 \pm 1.4 \mathrm{MeV}$ and $4320.0 \pm 10.4 \pm 7.0 \mathrm{MeV}$, and the former, regarded previously as $Y(4260)$, was renamed to $Y(4220)$. The $Y(4220)$ mass was confirmed by cross section measurements of $e^{+} e^{-} \rightarrow \omega \chi_{c 0}$ [8], $\pi^{+} \pi^{-} h_{c}$ [9], $\pi^{+} \pi^{-} \psi(3686)$ [10], and $D^{0} D^{*-} \pi^{+}$[11]. Currently, the known decays of $Y(4220)$ occur only to open or hidden charm final states. However, some theories argue that the charmonium-like states, such as $Y(4220)$, are very likely to also decay to light hadrons [12]. BESIII has reported measurements of the cross sections for the light hadron processes of $e^{+} e^{-} \rightarrow K_{S}^{0} K^{ \pm} \pi^{\mp} \pi^{0}(\eta)[13], K_{S}^{0} K^{ \pm} \pi^{\mp}[14]$, $p \bar{n} K_{S}^{0} K^{-}+$c.c. [15], and $p \bar{p} \pi^{0}$ [16], but no hint of charmless $Y(4220)$ decays have been found. Comprehensive measurements of the cross sections for $e^{+} e^{-} \rightarrow$ light hadrons are important to search for charmless decays of $Y$ states and to deeply explore the composition and properties of $Y$ states.

Searches for di-baryon or hexaquark states via $p p$ and $p n$ scattering processes have been carried out in fixed target experiments. A resonance $d^{*}(2380)$ in the isoscalar twopion fusion process $p n \rightarrow d \pi^{0} \pi^{0}$ was observed by WASA [17]. This state was later confirmed by the other two-pion fusion processes $p n \rightarrow d \pi^{+} \pi^{-}$[18] and $p p \rightarrow d \pi^{+} \pi^{0}$ [19], and the two-pion nonfusion processes $p n \rightarrow p p \pi^{0} \pi^{-}$[20] and $p n \rightarrow p n \pi^{0} \pi^{0}$ [21]. However, no experimental information is available in any $e^{+} e^{-}$collision experiment.

In the analysis presented in this paper, we study for the first time the $e^{+} e^{-} \rightarrow 2(p \bar{p})$ process in the center-of-mass (c.m.) energy $(\sqrt{s})$ region between 4.0 and $4.6 \mathrm{GeV}$. We search for the $Y(4220)$ structure by fitting the lineshape of the Born cross sections measured at these c.m. energies. In addition, we search for a potential structure similar to $d^{*}(2380)$ in the $p p$ and $\bar{p} \bar{p}$ mass spectra.

\section{THE BESIII DETECTOR AND DATA SAMPLES}

The BESIII detector is a magnetic spectrometer [22] located at the Beijing Electron Positron Collider (BEPCII). The cylindrical core of the BESIII detector consists of a helium-based multilayer drift chamber (MDC), a plastic scintillator time-of-flight (TOF) system, and a CsI(Tl) electromagnetic calorimeter (EMC), which are all enclosed in a superconducting solenoidal magnet providing a $1.0 \mathrm{~T}$ magnetic field. The solenoid is supported by an octagonal flux-return yoke with resistive plate counter muon identifier modules interleaved with steel. The acceptance of charged particles and photons is $93 \%$ over $4 \pi$ solid angle. The charged-particle momentum resolution at $1 \mathrm{GeV} / c$ is $0.5 \%$, and the specific ionization energy $\operatorname{loss}(\mathrm{d} E / \mathrm{d} x)$ resolution is $6 \%$ for the electrons from Bhabha scattering. The EMC measures photon energies with a resolution of $2.5 \%(5 \%)$ at $1 \mathrm{GeV}$ in the barrel (end cap) region. The time resolution of the TOF barrel part is $68 \mathrm{ps,} \mathrm{while} \mathrm{that} \mathrm{of} \mathrm{the}$ end cap part is 110 ps.

The 23 data sets taken at $\sqrt{s}=4.0-4.6 \mathrm{GeV}$ are used for this analysis. The nominal energy of each data set is calibrated by the di-muon process $e^{+} e^{-} \rightarrow\left(\gamma_{\mathrm{ISR} / \mathrm{FSR}}\right) \mu^{+} \mu^{-}$[23], where the subscript ISR/FSR stands for the initial-state or final-state radiative process, respectively. The integrated luminosity $\mathcal{L}$ is determined using large angle Bhabha events [24], and the total integrated luminosity is approximately $15 \mathrm{fb}^{-1}$.

The response of the BESIII detector is modeled with Monte Carlo (MC) simulations using the software framework BOOST [25] based on GEANT4 [26], which includes the geometry and material description of the BESIII detectors, the detector response and digitization models, as well as a database that keeps track of the running conditions and the detector performance. MC samples are used to optimize the selection criteria, evaluate the signal efficiency, and estimate backgrounds.

Inclusive MC samples are generated at different c.m. energies to study the potential backgrounds. The inclusive MC samples consist of the production of open-charm processes, the ISR production of vector charmonium and charmonium-like states, and the continuum processes incorporated in KKMC [27]. The known decay modes are modeled with EvtGen [28] using branching fractions taken from the Particle Data Group (PDG) [29], and the remaining unknown decays from the charmonium states with LundCharm [30]. The FSR from charged final-state particles are incorporated with Pнотоs [31]. The signal MC samples are generated with a phase-space (PHSP) distribution for the same 23 energy points as data.

\section{DATA ANALYSIS}

For each candidate event, it is required that there are four good charged tracks. Two of them must be identified as 


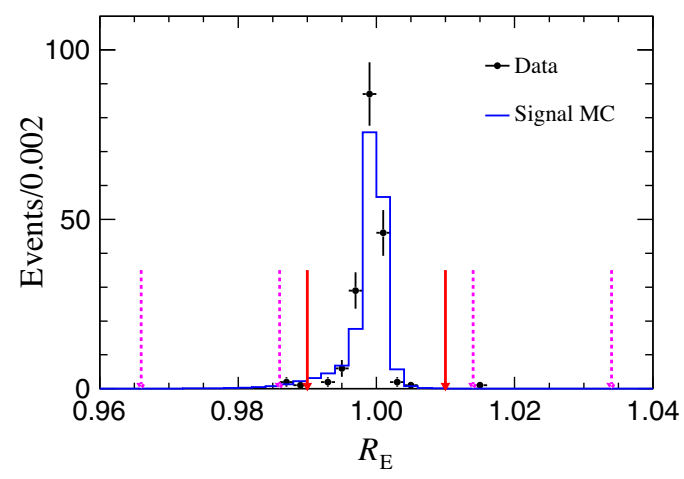

FIG. 1. Distributions of $R_{E}$ of the accepted candidate events in data (dots with error bars) and signal MC simulation (histogram) from all c.m. energies. The region between the two solid red arrows is the signal region, while the region between the two dashed pink arrows is the sideband region.

protons and two as antiprotons. The charged particles are required to be within the acceptance range of $|\cos \theta|<0.93$, where $\theta$ is the polar angle with respect to the MDC axis. All the charged tracks are required to originate from the interaction region $R_{x y}<1 \mathrm{~cm}$ and $\left|V_{z}\right|<10 \mathrm{~cm}$, where $R_{x y}$ and $\left|V_{z}\right|$ are the distances of closest approach of the charged track to the interaction point in the $x y$-plane and $z$ direction, respectively. For particle identification (PID), the $\mathrm{d} E / \mathrm{d} x$ measured by the MDC and the TOF information are used to calculate the confidence levels for the particle hypotheses of pion, kaon, and proton. If the confidence level for the proton (antiproton) hypothesis is larger than that for the other two hypotheses, it is identified as a proton (antiproton). The efficiency of PID is $80 \%-100 \%$ as a function of transverse momentum of proton (antiproton).

A three-constraint (3C) kinematic fit imposing threemomentum conservation under the hypothesis of $e^{+} e^{-} \rightarrow$ $2(p \bar{p})$ is performed for the four candidate charged tracks to suppress background events. Since the energy will be used in determining the signal yield, it is not constrained in the kinematic fit. The candidate events with $\chi_{3 \mathrm{C}}^{2}<60$ are kept for further analysis.

The signal yield is determined by a kinematic variable $R_{E}=E_{\text {measure }} / E_{\mathrm{cm}}$, where $E_{\text {measure }}$ is the total energy of all final particles and $E_{\mathrm{cm}}$ is the c.m. energy. Figure 1 shows the $R_{E}$ distribution of the accepted candidate events. The signal events concentrate around 1.0 in the $R_{E}$ distribution. The signal region is defined as the region with $R_{E} \in(0.99,1.01)$, while the sideband region is defined as the region with $R_{E} \in(0.966,0.986) \cup(1.014,1.034)$.

TABLE I. The integrated luminosities $(\mathcal{L})$, detection efficiencies $(\epsilon)$, radiative correction factors $\left(1+\delta^{\gamma}\right)$, vacuum polarization factors $\left(1+\delta^{\nu}\right)$, and the Born cross section $\left(\sigma^{\mathrm{B}}\right)$ at different c.m. energies $(\sqrt{s})$. The first uncertainties for cross sections are statistical and the second are systematic, respectively, while those for $N^{\text {obs }}, N^{\text {bkg }}, N^{\text {net }}$, and $\epsilon$ are statistical only.

\begin{tabular}{|c|c|c|c|c|c|c|c|c|}
\hline$\sqrt{s}(\mathrm{GeV})$ & $N_{\text {data }}^{\text {obs }}$ & $N_{\text {data }}^{\mathrm{bkg}}$ & $N_{\text {data }}^{\text {net }}$ & $\mathcal{L}\left(\mathrm{pb}^{-1}\right)$ & $\epsilon(\%)$ & $\left(1+\delta^{\gamma}\right)$ & $\frac{1}{|1-\Pi|^{2}}$ & $\sigma^{\text {Born }}(\mathrm{fb})$ \\
\hline 4.009 & $1.0_{-0.8}^{+2.3}$ & $0.0_{-0.0}^{+0.5}$ & $1.0_{-0.8}^{+2.4}$ & 482.0 & $13.6 \pm 0.1$ & 0.8211 & 1.0441 & $18_{-14}^{+43} \pm 2$ \\
\hline 4.160 & $2.0_{-1.3}^{+2.8}$ & $0.0_{-0.0}^{+0.0}$ & $2.0_{-1.3}^{+2.8}$ & 406.9 & $26.0 \pm 0.1$ & 0.8492 & 1.0533 & $21_{-14}^{+29} \pm 2$ \\
\hline 4.180 & $16.0_{-4.0}^{+5.1}$ & $0.0_{-0.0}^{+0.0}$ & $16.0_{-4.0}^{+5.1}$ & 3194.5 & $28.6 \pm 0.1$ & 0.8502 & 1.0541 & $20_{-5}^{+6} \pm 2$ \\
\hline 4.190 & $10.0_{-3.1}^{+4.3}$ & $0.0_{-0.0}^{+0.5}$ & $10.0_{-3.1}^{+4.3}$ & 523.9 & $29.3 \pm 0.1$ & 0.8503 & 1.0558 & $73_{-22}^{+31} \pm 6$ \\
\hline 4.200 & $5.0_{-2.2}^{+3.4}$ & $0.0_{-0.0}^{+0.0}$ & $5.0_{-2.2}^{+3.1}$ & 525.2 & $30.0 \pm 0.1$ & 0.8515 & 1.0565 & $35_{-16}^{+24} \pm 3$ \\
\hline 4.210 & $1.0_{-0.8}^{+2.3}$ & $0.5_{-0.4}^{+1.0}$ & $0.5_{-0.9}^{+2.6}$ & 517.2 & $30.7 \pm 0.1$ & 0.8522 & 1.0568 & $3_{-6}^{+18} \pm 1$ \\
\hline 4.220 & $8.0_{-2.8}^{+4.0}$ & $0.0_{-0.0}^{+0.5}$ & $8.0_{-2.8}^{+4.0}$ & 513.4 & $31.4 \pm 0.1$ & 0.8515 & 1.0563 & $55_{-19}^{+28} \pm 4$ \\
\hline 4.230 & $12.0_{-3.4}^{+4.6}$ & $0.0_{-0.0}^{+0.0}$ & $12.0_{-3.4}^{+4.6}$ & 1056.4 & $32.1 \pm 0.1$ & 0.8529 & 1.0564 & $39_{-11}^{+15} \pm 3$ \\
\hline 4.237 & $8.0_{-2.8}^{+4.0}$ & $0.0_{-0.0}^{+0.5}$ & $8.0_{-2.8}^{+4.0}$ & 529.1 & $32.5 \pm 0.1$ & 0.8527 & 1.0555 & $52_{-18}^{+26} \pm 4$ \\
\hline 4.246 & $3.0_{-1.6}^{+2.9}$ & $0.0_{-0.0}^{+0.5}$ & $3.0_{-1.6}^{+3.0}$ & 536.3 & $33.1 \pm 0.1$ & 0.8535 & 1.0555 & $19_{-10}^{+19} \pm 2$ \\
\hline 4.260 & $\begin{array}{l}5.0_{-2.2}^{+3.4} \\
\text {. }\end{array}$ & $0.0_{-0.0}^{+0.5}$ & $5.0_{-2.2}^{+3.4}$ & 828.4 & $33.9 \pm 0.1$ & 0.8543 & 1.0536 & $20_{-9}^{+13} \pm 2$ \\
\hline 4.270 & $2.0_{-1.3}^{+2.6}$ & $0.0_{-0.0}^{+0.0}$ & $2.0_{-1.3}^{+2.2}$ & 529.7 & $34.5 \pm 0.2$ & 0.8545 & 1.0530 & $12_{-8}^{+16} \pm 1$ \\
\hline 4.280 & $7.0_{-2.6}^{+3.8}$ & $0.0_{-0.0}^{+0.0}$ & $7.0_{-2.6}^{+3.8}$ & 175.2 & $35.1 \pm 0.2$ & 0.8545 & 1.0530 & $126_{-47}^{+69} \pm 9$ \\
\hline 4.290 & $5.0_{-2.2}^{+3.4}$ & $0.0_{-0.0}^{+0.5}$ & $5.0_{-2.2}^{+3.4}$ & 491.5 & $33.7 \pm 0.1$ & 0.8541 & 1.0527 & $34_{-15}^{+23} \pm 3$ \\
\hline 4.315 & $4.0_{-1.9}^{+3.2}$ & $0.0_{-0.0}^{+0.0}$ & $4.0_{-1.9}^{+3.2}$ & 492.1 & $35.0 \pm 0.2$ & 0.8554 & 1.0522 & $26_{-12}^{+21} \pm 2$ \\
\hline 4.340 & $6.0_{-2.4}^{+3.6}$ & $0.0_{-0.0}^{+0.0}$ & $6.0_{-2.4}^{+3.6}$ & 501.1 & $36.2 \pm 0.2$ & 0.8557 & 1.0508 & $37_{-15}^{+22} \pm 3$ \\
\hline 4.360 & $4.0_{-1.9}^{+3.2}$ & $0.0_{-0.0}^{+0.5}$ & $4.0_{-1.9}^{+3.2}$ & 543.9 & $39.2 \pm 0.2$ & 0.8556 & 1.0511 & $21_{-10}^{+17} \pm 2$ \\
\hline 4.380 & $8.0_{-2.8}^{+4.0}$ & $0.0_{-0.0}^{+0.5}$ & $8.0_{-2.8}^{+4.0}$ & 522.8 & $38.0 \pm 0.2$ & 0.8560 & 1.0513 & $45_{-16}^{+22} \pm 4$ \\
\hline 4.400 & $5.0_{-2.2}^{+3.4}$ & $0.0_{-0.0}^{+0.0}$ & $5.0_{-2.2}^{+3.4}$ & 505.0 & $38.9 \pm 0.2$ & 0.8558 & 1.0510 & $28_{-12}^{+19} \pm 3$ \\
\hline 4.420 & $16.0_{-4.0}^{+5.1}$ & $0.0_{-0.0}^{+0.5}$ & $16.0_{-4.0}^{+5.1}$ & 1043.9 & $39.7 \pm 0.2$ & 0.8552 & 1.0524 & $43_{-11}^{+14} \pm 4$ \\
\hline 4.440 & $17.0_{-4.1}^{+4.0}$ & $0.0_{-0.0}^{+0.0}$ & $17.0_{-4.1}^{+4.0}$ & 568.1 & $40.4 \pm 0.2$ & 0.8548 & 1.0537 & $82_{-20}^{+25} \pm 6$ \\
\hline 4.470 & $4.0_{-1.9}^{+3.1}$ & $0.0_{-0.0}^{+0.0}$ & $4.0_{-1.9}^{+3.1}$ & 111.1 & $41.5 \pm 0.2$ & 0.8547 & 1.0548 & $96_{-46}^{+77} \pm 7$ \\
\hline 4.600 & $24.0_{-4.9}^{+6.0}$ & $0.0_{-0.0}^{+0.5}$ & $24.0_{-4.9}^{+6.0}$ & 586.9 & $45.0 \pm 0.2$ & 0.8551 & 1.0546 & $101_{-21}^{+25} \pm 8$ \\
\hline
\end{tabular}



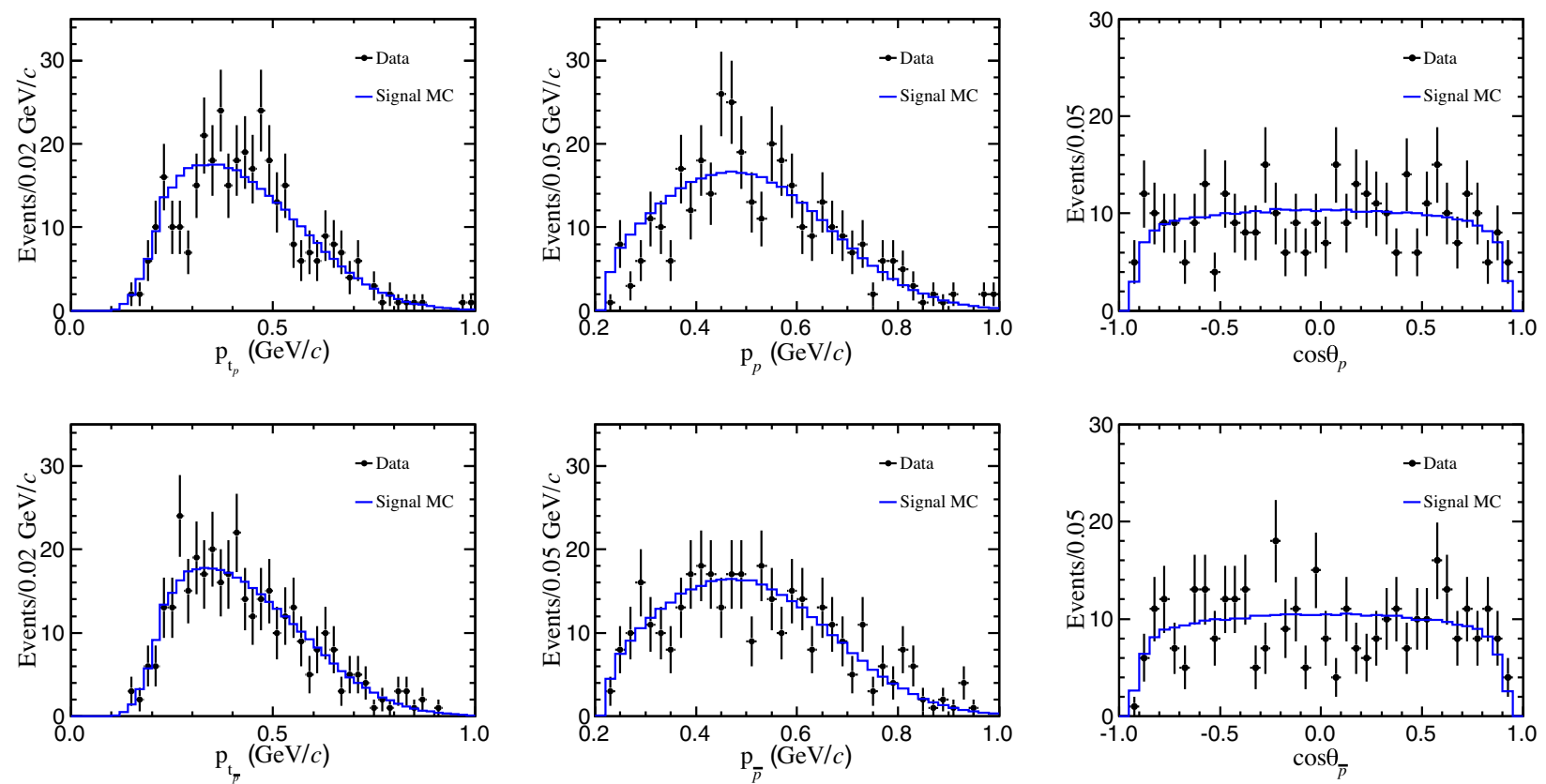

FIG. 2. Transverse-momentum distribution (left) and the momentum distribution (middle), the polar-angle distribution (right) for the proton (top) and antiproton (bottom) for all data (filled circles with error bars), and signal MC simulation (histogram). Please note that there are two entries for each event.
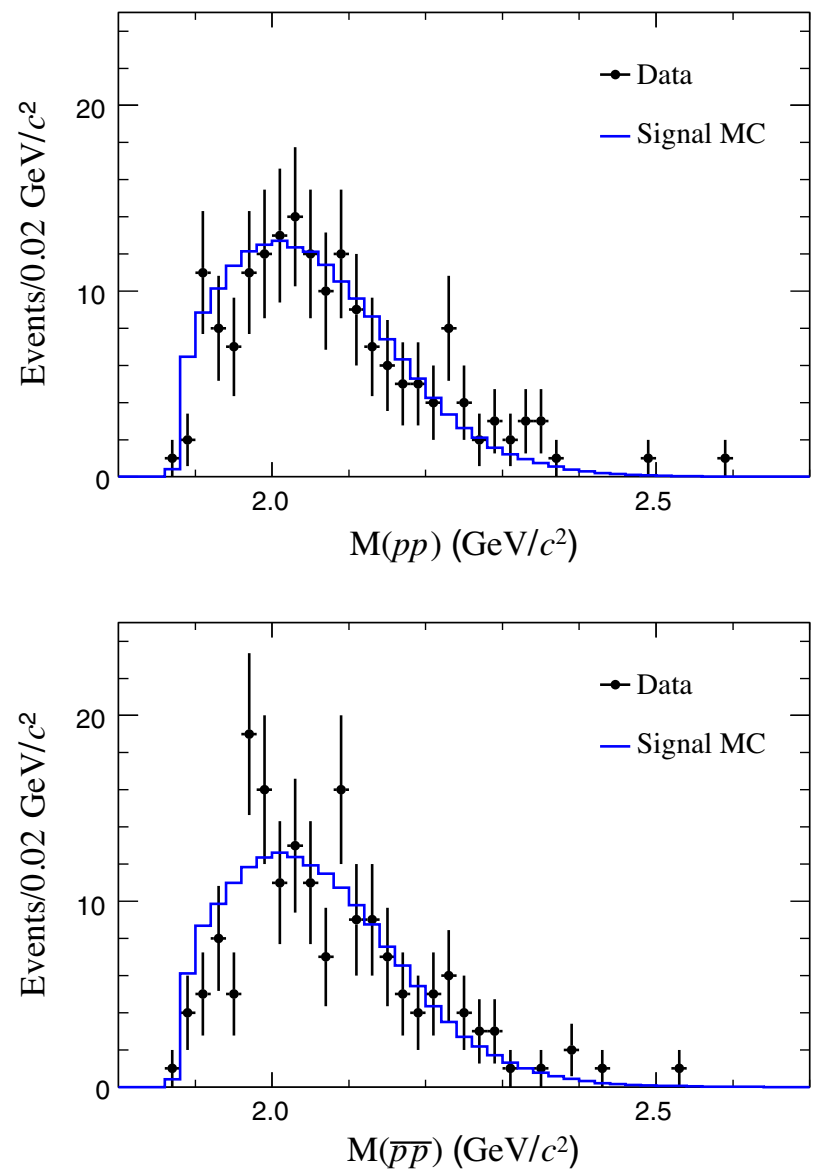

FIG. 3. Invariant-mass distributions of $p p$ (top) and $\bar{p} \bar{p}$ (bottom) from all data (filled circles with error bars) and signal MC simulation (histogram).
Studies based on the inclusive MC samples show that only a few background events survive at $4.180 \mathrm{GeV}$, and they do not form a peak. The background in the $R_{E}$ signal region is estimated by the events in the $R_{E}$ sideband region multiplied by a scale factor of 0.5 assuming that the background is flat. The numbers of events in the $R_{E}$ signal region in data and the scaled background yields, which are obtained by counting, are summarized in the third and fourth columns of Table I.

\section{DETECTION EFFICIENCY}

Figure 2 compares momenta, transverse momenta, and polar angle distributions between accepted candidate events of data and signal MC samples. Throughout the paper, the data and $\mathrm{MC}$ distributions sum over all c.m. energies unless stated otherwise, while the MC distributions have been weighted by the data signal yields. There is good agreement between data and MC simulation. Therefore, the MC events generated according to PHSP are used to determine the detection efficiency. The $p p$ and $\bar{p} \bar{p}$ invariant-mass spectra are shown in Fig. 3, and no obvious structure is found.

The Born cross section of $e^{+} e^{-} \rightarrow 2(p \bar{p})$ at each c.m. energy is calculated as

$$
\sigma^{\text {Born }}=\frac{N^{\text {net }}}{\mathcal{L} \times \epsilon \times\left(1+\delta^{\gamma}\right) \times \frac{1}{|1-\Pi|^{2}}},
$$

where $N^{\text {net }}$ is the net number of signal events after 
background subtraction, $\mathcal{L}$ is the integrated luminosity of the data set, $\epsilon$ is the detection efficiency, $\left(1+\delta^{\gamma}\right)$ and $\frac{1}{|1-\Pi|^{2}}$ are the ISR and vacuum polarization correction factors, respectively.

To obtain $\left(1+\delta^{\gamma}\right)$ and $\frac{1}{|1-\Pi|^{2}}$, we take the cross section according to the energy-dependent lineshape of $1 / s$ as the initial input, and obtain the Born cross section by iteration until the efficiencies become stable at all energies. The difference of $\epsilon \cdot\left(1+\delta^{\gamma}\right)$ between the last two iterations is required to be less than $1 \%$. The relevant numbers related to Born cross section measurement are summarized in Table I.

\section{SYSTEMATIC UNCERTAINTY}

The uncertainty in the measurement of the integrated luminosity of the data set is $1.0 \%$ [24]. The uncertainties of the tracking and PID efficiencies have been studied with high purity control samples of $J / \psi \rightarrow p \bar{p} \pi^{+} \pi^{-}$and $\psi(3686) \rightarrow \pi^{+} \pi^{-} J / \psi \rightarrow \pi^{+} \pi^{-} p \bar{p}[32,33]$. The differences of the tracking and PID efficiencies between data and MC simulation in different transverse momentum and momentum ranges are obtained separately. The averaged differences for tracking (PID) efficiencies that are reweighted by the proton and antiproton momenta of the signal MC events, $0.5 \%$ (1.3\%) per proton and $1.0 \%$ (1.8\%) per antiproton, are assigned as the tracking (PID) systematic uncertainties. Adding them linearly gives our estimate of the total systematic uncertainty of the tracking (PID) efficiency for all charged tracks resulting in 3.0\% (6.2\%).

To determine the systematic error related to uncertainties in the signal window, we define a ratio which is the number of net signal events in the signal window obtained by counting to the number of signal events obtained by fitting to the $R_{E}$ spectra. For data, the combined $R_{E}$ spectra are fitted with a MC-derived shape convolved with a Gaussian to describe the signal shape, while the background shape is described by a first-order Chebychev polynomial. For MCsimulated data at each energy point, the ratio is similarly obtained. The relative differences of the ratio between data and $\mathrm{MC}$ simulations range from $0.5 \%$ to $1.4 \%$, depending on the energy point, and are taken as the uncertainties.

To obtain reliable detection efficiencies, the Born cross sections input in the generator have been iterated until the $\left(1+\delta^{r}\right) \cdot \epsilon$ values converge. The differences of $\left(1+\delta^{r}\right) \cdot \epsilon$ between the last two iterations in the cross section measurements, which range from $0.0 \%$ to $1.0 \%$, are taken as the systematic uncertainties due to the ISR correction factor.

The systematic uncertainty from the kinematic fit is estimated by changing the $\chi_{3 \mathrm{C}}^{2}$ requirement by \pm 15 . The largest changes of the cross sections compared to the nominal $\chi_{3 \mathrm{C}}^{2}$ requirement range from $0.3 \%$ to $0.7 \%$ and are taken as the corresponding uncertainties. The total systematic uncertainty is determined to range from $7.0 \%$ to
$7.1 \%$, by summing the individual values in quadrature under the assumption that all the sources are independent.

\section{FIT TO THE BORN CROSS SECTIONS}

We fit to the Born cross sections under four assumptions with the least-square method [34]. In order to describe purely continuum production, the first cases are based on a simple four-body energy-dependent PHSP lineshape [35]. The second case is based on an exponential function $[36,37]$. The exponential function is constructed as

$$
\sigma^{\mathrm{Born}}(s)=\frac{1}{s} \times e^{-p_{0}\left(\sqrt{s}-M_{\mathrm{th}}\right)} \times p_{1},
$$

where $p_{0}$ and $p_{1}$ are free parameters, and $M_{\text {th }}$ is $\left(2 m_{p}+2 m_{\bar{p}}\right)$. The third (fourth) case is based on an exponential function for continuum production plus the well-established charmonium state $\psi(4160)$ [charmoniumlike state $Y(4220)]$ for resonance production. For the latter two cases, the light-hadron production is described as

$$
\sigma^{\mathrm{Born}}(s)=\left|\mathrm{BW}(\sqrt{s}) e^{i \phi}+\sqrt{f(\sqrt{s})}\right|^{2},
$$

where $\sqrt{f(\sqrt{s})}$ denotes the chosen continuum production amplitude, the resonance amplitude is described by a relativistic Breit-Wigner amplitude $\mathrm{BW}(\sqrt{s})=\frac{\sqrt{12 \pi \Gamma^{\mathrm{ee}} \Gamma^{\mathrm{tot}}}}{s-\mathrm{M}^{2}+i \mathrm{M} \Gamma^{\mathrm{tot}}}$, and $\phi$ is the phase angle between the two amplitudes. Moreover, $\mathrm{M}, \Gamma^{e e}$, and $\Gamma^{\text {tot }}$ are the mass, partial width to $e^{+} e^{-}$and total width of the assumed resonance, and the values are taken from the PDG [29], which are $\mathrm{M}=$ 4.191(4.23) GeV/c $c^{2}$ and $\Gamma^{\text {tot }}=70(55) \mathrm{MeV}$ for $\psi(4160)[Y(4220)]$. The fit results are shown in Fig. 4.

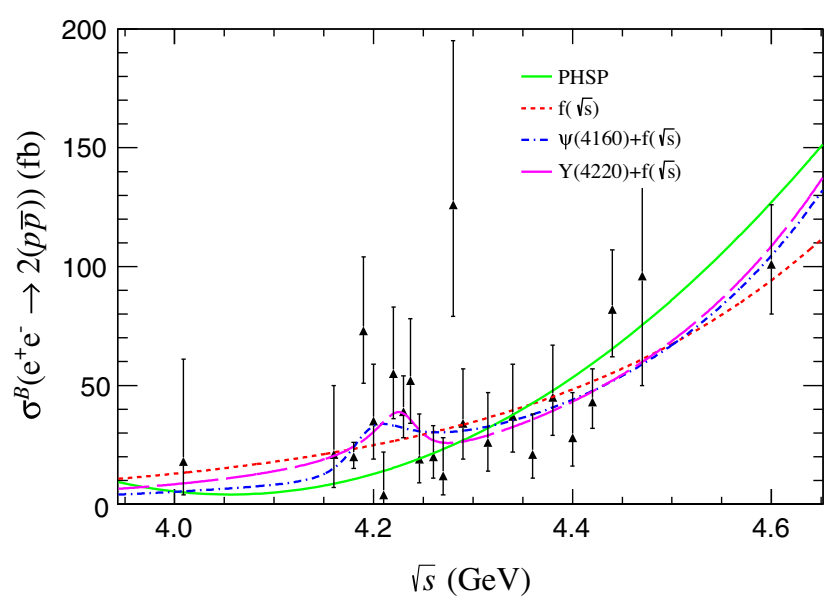

FIG. 4. Born cross sections of the process $e^{+} e^{-} \rightarrow 2(p \bar{p})$ as a function of c.m. energy. The data are presented as filled triangles with error bars corresponding to the combination of statistical and systematic uncertainties. The lines are fit results to various model assumptions which are described in the text. 
The goodness-of-fit is $\chi^{2} / \mathrm{NDF}=1.9,1.2,1.3$, and 1.2 for the four fit scenarios, respectively. Here, NDF is the number of degrees of freedom. The large goodness-of-fit for the first case implies that it is less preferable to describe the $e^{+} e^{-} \rightarrow 2(p \bar{p})$ process by a simple four-body energydependent PHSP assumption, while it can be by the exponential function. The statistical significance of the resonances is estimated by comparing the change of $\chi^{2}$ with and without the resonance and taking the change of degrees of freedom into account. The statistical significance is $0.83 \sigma(1.69 \sigma)$ for $\psi(4160)[Y(4220)]$, which indicates it is unnecessary to include the $\psi(4160)$ or $Y(4220)$ resonance. This could also imply that the $\psi(4160)$ and $Y(4220)$ disfavor a decay to $2(p \bar{p})$.

\section{SUMMARY}

In conclusion, the process of $e^{+} e^{-} \rightarrow 2(p \bar{p})$ is studied at $23 \mathrm{c.m}$. energies in the region from 4.0 to $4.6 \mathrm{GeV}$. The Born cross sections at the different c.m. energies are measured, and the lineshape can be generally described by an empirical exponential function. The significances for possible contributions by a $\psi(4160)$ or $Y(4220)$ resonance are small, namely $0.83 \sigma$ and $1.69 \sigma$, respectively. With the present statistics it is impossible to draw any conclusion as to whether there are actual resonances or structures in this lineshape.

The baryon-pair invariant-mass spectra are in good agreement with phase space, and no hexaquark or dibaryon state is found with the currently available statistics.

\section{ACKNOWLEDGMENTS}

The BESIII collaboration thanks the staff of BEPCII and the IHEP computing center for their strong support. This work is supported in part by National Key Basic Research Program of China under Contract No. 2015CB856700; National Natural Science Foundation of China (NSFC) under Contracts Nos. 11975118, 11625523, 11635010 , 11735014, 11822506, 11835012, 11935015, 11935016, 11935018, 11961141012; the Natural Science Foundation of Hunan Province under Contract No. 2019JJ30019; the Chinese Academy of Sciences (CAS) Large-Scale Scientific Facility Program; Joint Large-Scale Scientific Facility Funds of the NSFC and CAS under Contracts Nos. U1732263, U1832207; CAS Key Research Program of Frontier Sciences under Contracts Nos. QYZDJ-SSWSLH003, QYZDJ-SSW-SLH040; 100 Talents Program of CAS; Institute of Nuclear and Particle Physics, Astronomy and Cosmology (INPAC) and Shanghai Key Laboratory for Particle Physics and Cosmology; ERC under Contract No. 758462; German Research Foundation DFG under Contracts Nos. Collaborative Research Center CRC 1044, FOR 2359; Istituto Nazionale di Fisica Nucleare, Italy; Ministry of Development of Turkey under Contract No. DPT2006K-120470; National Science and Technology fund; STFC (United Kingdom); The Knut and Alice Wallenberg Foundation (Sweden) under Contract No. 2016.0157; The Royal Society, UK under Contracts Nos. DH140054, DH160214; The Swedish Research Council; U.S. Department of Energy under Contracts Nos. DE-FG02-05ER41374, DE-SC-0012069.
[1] S. K. Choi et al. (Belle Collaboration), Phys. Rev. Lett. 91, 262001 (2003).

[2] B. Aubert et al. (BABAR Collaboration), Phys. Rev. Lett. 95, 142001 (2005).

[3] M. Ablikim et al. (BESIII Collaboration), Phys. Rev. Lett. 110, 252001 (2013).

[4] Z. Q. Liu et al. (Belle Collaboration), Phys. Rev. Lett. 110, 252002 (2013).

[5] T. E. Coan et al. (CLEO Collaboration), Phys. Rev. Lett. 96, 162003 (2006).

[6] C. Z. Yuan et al. (Belle Collaboration), Phys. Rev. Lett. 99, 182004 (2007).

[7] M. Ablikim et al. (BESIII Collaboration), Phys. Rev. Lett. 118, 092001 (2017).

[8] M. Ablikim et al. (BESIII Collaboration), Phys. Rev. Lett. 114, 092003 (2015).

[9] M. Ablikim et al. (BESIII Collaboration), Phys. Rev. Lett. 118, 092002 (2017).

[10] M. Ablikim et al. (BESIII Collaboration), Phys. Rev. D 96, 032004 (2017).
[11] M. Ablikim et al. (BESIII Collaboration), Phys. Rev. Lett. 122, 102002 (2019).

[12] S. Dubynskiy and M. B. Voloshin, Phys. Lett. B 666, 344 (2008).

[13] M. Ablikim et al. (BESIII Collaboration), Phys. Rev. D 99, 012003 (2019).

[14] M. Ablikim et al. (BESIII Collaboration), Phys. Rev. D 99, 072005 (2019).

[15] M. Ablikim et al. (BESIII Collaboration), Phys. Rev. D 98, 032014 (2018).

[16] M. Ablikim et al. (BESIII Collaboration), Phys. Lett. B 771, 45 (2017).

[17] M. Bashkanov et al. (CELSIUS/WASA Collaboration), Phys. Rev. Lett. 102, 052301 (2009); P. Adlarson et al. (WASA-at-COSY Collaboration), Phys. Rev. Lett. 106, 242302 (2011).

[18] F. Kren et al. (CELSIUS/WASA Collaboration), Phys. Lett. B 684, 110 (2010); 702, 312(E) (2011).

[19] P. Adlarson et al. (WASA-at-COSY Collaboration), Phys. Rev. Lett. 106, 242302 (2011). 
[20] P. Adlarson et al. (WASA-at-COSY Collaboration), Phys. Rev. C 88, 055208 (2013).

[21] P. Adlarson et al. (WASA-at-COSY Collaboration), Phys. Lett. B 743, 325 (2015).

[22] M. Ablikim et al. (BESIII Collaboration), Nucl. Instrum. Methods Phys. Res., Sect. A 614, 345 (2010).

[23] M. Ablikim et al. (BESIII Collaboration), Chin. Phys. C 40, 063001 (2016).

[24] M. Ablikim et al. (BESIII Collaboration), Chin. Phys. C 39, 093001 (2015).

[25] Z. Y. Deng et al., High Energy Phys. Nucl. Phys. 30, 371 (2006).

[26] S. Agostinelli et al. (GEANT4 Collaboration), Nucl. Instrum. Methods Phys. Res., Sect. A 506, 250 (2003).

[27] S. Jadach, B. F. L. Ward, and Z. Was, Phys. Rev. D 63, 113009 (2001); Comput. Phys. Commun. 130, 260 (2000).

[28] R. G. Ping, Chin. Phys. C 32, 599 (2008); D. J. Lange, Nucl. Instrum. Methods Phys. Res., Sect. A 462, 152 (2001).
[29] M. Tanabashi et al. (Particle Data Group), Prog. Theor. Exp. Phys. 2020, 083C01 (2020).

[30] J. C. Chen, G. S. Huang, X. R. Qi, D. H. Zhang, and Y. S. Zhu, Phys. Rev. D 62, 034003 (2000); R. L. Yang, R. G. Ping, and D. Chen, Chin. Phys. Lett. 31, 061301 (2014).

[31] E. Richter-Was, Phys. Lett. B 303, 163 (1993).

[32] M. Ablikim et al. (BESIII Collaboration), Phys. Rev. D 91, 112004 (2015).

[33] M. Ablikim et al. (BESIII Collaboration), Phys. Rev. D 99, 031101 (2019).

[34] B. P. Roe, Probability and Statistics in Experimental Physics, 2nd ed. (Springer-Verlag, New York, 2001).

[35] E. Byckling and K. Kajantie, Particle Kinematics (Wiley, New York, 1973).

[36] J. P. Lees et al. (BABAR Collaboration), Phys. Rev. D 86, 051102(R) (2012).

[37] M. Ablikim et al. (BESIII Collaboration), Phys. Rev. Lett. 118, 092001 (2017). 